\title{
Annular elastolytic giant cell granuloma: clinical case and literature review
}

\begin{abstract}
Annular elastolytic giant cell granuloma (AEGCG) is rare granulomatous dermatoses of uncertain pathogenesis. It usually presents itself in adults over the age of 40 Clinically characterized by annular plaques with well defined erythematous borders with a normal or atrophic center in a polycyclical pattern which usually presents itself in photoexposed areas of the skin. It is necessary to have histological study of the lesions for the diagnosis. In which you can see a granulomatous reaction with elastolysis, phagocytosis of elastic fibers and multinucleated giant cells with absence or reduction of elastic fibers. We will present a case of a 73 year old woman with AEGCG with the main differential diagnosis being discoid lupus.
\end{abstract}

Volume 5 Issue 5 - 2018

\begin{abstract}
Nicolás Hermosilla,' Alexis Ramos, ${ }^{2}$ Álvaro Fierro, ${ }^{2}$ Carmen Varas ${ }^{3}$

'Doctor of Medicine, Hospital San Pablo de Coquimbo, Chile ${ }^{2}$ Student of Medicine, Pontificia Universidad Católica de Chile, Chile

${ }^{3}$ Dermatologist, Hospital San Pablo de Coquimbo, Chile
\end{abstract}

Correspondence: Nicolás Hermosilla, Doctor of Medicine, Hospital San Pablo de Coquimbo, Chile, Email nehermosilla@uc.cl

Received: July 23, 2018 | Published: September 21, 2018

\section{Introduction}

This entity was first described in 1975 by O'Brien, referring to it as a dermatoses characterized by papules or annular plaques, smooth borders, without scales, with a slightly atrophic hypopigmented center, postulating sun damage as the main etiopathogenic factor. $\mathrm{He}$ originally named it actinic granuloma (AG). Microscopically, it is seen as an infiltrate composed mainly of giant foreign body cells in relation to elastic fibers with little or no mucin, no necrobiosis or palisaded granuloma. ${ }^{1,2}$

Later, in 1979, Hanke described 5 cases of chronic inflammatory reactions consisting of annular plaques in non photo exposed areas of the skin, proposing the term Annular elastolytic giant cell granuloma. Contrary to O'Brien, Hanke declares that the destruction of the elastic fibers is a secondary and not a consequence of sun damage.

The actinic AG reported by O'Brien and the AEGCG described by Hanke show similar histological features of granulomatous inflammation with elastophagocytosis, for which some authors consider that actinic AG is not an entity of its own, but rather a variety of AECG in photo exposed areas. ${ }^{3,4}$

\section{Clinical case}

Female patient, 73 years old with a history of hypothyroidism in control. She consults for skin eruption in the posterior neck area (Figure 1), cleavage and forearms, asymptomatic. On physical examination, she presented papules and annular erythematous plaques, some of them in polycyclic distribution, in the areas described by the patient. A photosensitivity study is requested given the distribution of the lesions: Hemogram, HSV and normal biochemical profile. ANA (+) 1/160 fine speckled pattern, anti- dsDNA (-), ENA panel (-), normal CK levels, normal IgE levels and ASO (-). Under the suspicion of discoid lupus erythematosus a biopsy is performed, which reports: Presence of granuloma in the reticular dermis. There are giant cells associated with some hyaline fascicles and mild lymphocytic infiltrate (Figure 2). In one of the giant cells there are small elastotic fibers in the cytoplasm. With this results annular elastolytic giant cell granuloma is diagnosed. Topical $0.05 \%$ clobetasol is indicated, in a $10 \times 10$ schemes and photoprotection. The patient attends control 3 months later, observing a clear remission of the lesions.

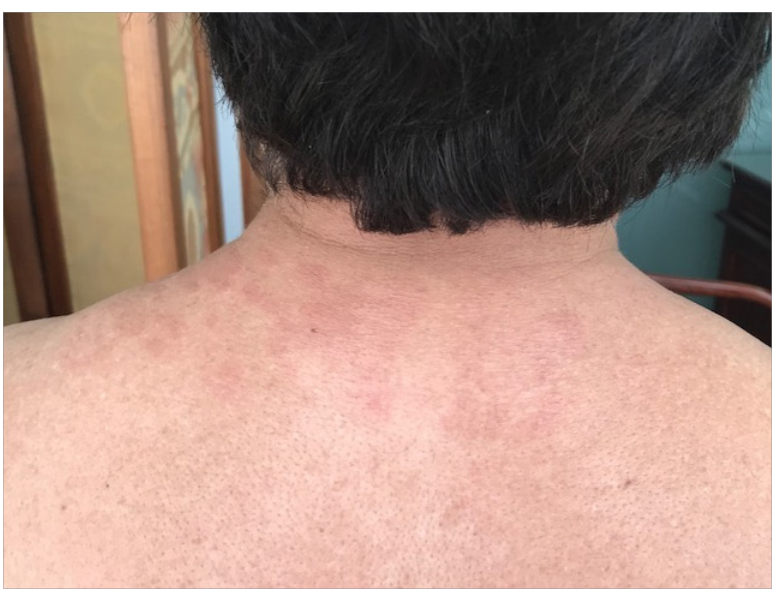

Figure I Erythematous annular plaque and papules, in a polycyclic pattern.

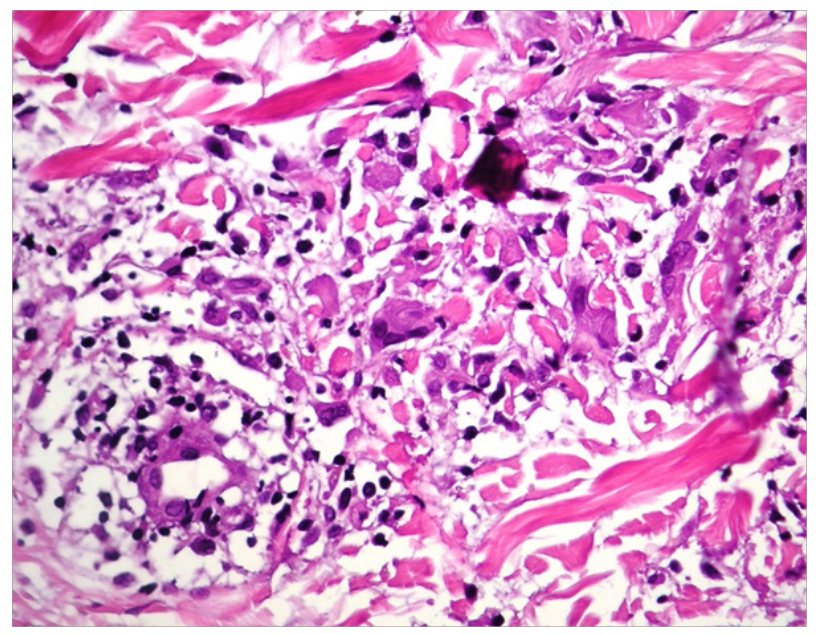

Figure 2 Granuloma in the reticular dermis. There are giant cells associated with some hyaline fascicles and mild lymphocytic infiltrate.

\section{Discussion}

AEGCG is an uncommon clinical variety of annular granuloma. This disease mainly presents itself from the fourth decade of life. Its 
appearance in some series has been reported mainly in the female gender, in other series in the male gender and in others even without difference between them. ${ }^{3,5-7}$

The etiopathogenesis of the AEGCG remains uncertain. It is postulated that ultraviolet radiation, heat, chronic use of drugs such as doxycycline and tanning beds used to obtain an artificial tan induce an immunological cell reaction where the immunopathogenicity of the elastic fiber plays a central role; apparently its antigenic capacity changes which induces the formation of an elastolytic granuloma resulting in the destruction of the elastic fibers. From the biomolecular point of view it is thought that the $67-\mathrm{kDa}$ elastin receptor and the metalloelastase- 12 play a role in its pathogenesis, but the molecular mechanism still remains to be fully elucidated. ${ }^{3,4}$

Clinically, classic AEGCG presents with a well-defined erythematous annular plaque or papule, in a polycyclic pattern, with pearly smooth borders and central atrophy. There can be multiple or unique lesions, which usually do not flake. The lesions initially present as papules, which progress in a centrifugal manner, causing annular, rounded, oval or polycyclic forms. The border is either erythematous or erythematous-violaceous and the central zone folded, atrophic, redundant, as a consequence of the lack of elastic fibers. The size of the lesions are usually bigger than $3 \mathrm{~cm}$, and are usually located in photo exposed areas such as the face, neck, arms and cleavage, but can also appear in covered areas. The plaques may be asymptomatic or cause mild pruritus, and may also compromise more than one area of the body; there are case reports of generalized presentation with extensive plaques. The disease predominates in the middle age of life and the clinical evolution is chronic in general, with a low tendency to spontaneous involution. ${ }^{3-5}$ There have been reported association of the AEGCG with other systemic diseases such as diabetes mellitus, temporal arteritis and several types of malignant entities including myeloid leukemia, sarcoidosis and monoclonal gammopathies. ${ }^{3,8}$

For the diagnosis it is necessary to study the lesions through biopsy. The histopathological study findings vary between the area analyzed, in the borders, numerous granulomas and giant cells, elastolysis and elastophagocytosis. In the center, few granulomas and a decrease or lack of elastic fibers. It is uncommon to observe sun damage in the papillary dermis and the epidermis is undamaged. The participating inflammatory cells are histiocytes, lymphocytes and few neutrophils, with little tendency to form palisading granuloma, nor appreciable mucin deposits. There are publications of extended, serpiginous, papular, reticular or exanthematic cases. ${ }^{4}$

The differential diagnosis includes granuloma annulare, secondary syphilis, lupus vulgaris, discoid lupus erythematosus, lichen annularis, lichen planus, and sarcoidosis. Treatment options are based on case reports ${ }^{9}$ and include numerous therapies such as topical steroids, topical pimecrolimus, excision of lesions, cryotherapy, ultraviolet A therapy with psoralen, systemic clofazimine, chloroquine / oral hydroxychloroquine, cyclosporine, systemic corticosteroids, fumaric acid, dapsone, tranilast and isotretinoin, with responses that vary according to the report. ${ }^{3}$ The most frequently used modalities include topical corticosteroids and immunomodulators, intralesional corticosteroids and cryotherapy. Systemic medications with antimalarials, oral retinoids, systemic corticosteroids, and cyclosporin are usually considered in resistant cases. ${ }^{8,9}$

\section{Acknowledgements}

None.

\section{Conflict of interest}

Author declares that there is no conflict of interest.

\section{References}

1. O'Brien JP. Actinic granuloma. An annular connective tissue disorder affecting sun- and heat-damaged (elastotic) skin. Arch Dermatol. 1975;111(4):460-466.

2. Hanke CW, Bailin PL, Roenigk HH Jr. Annular elastolytic giant cell granuloma. A clinicopathologic study of five cases and a review of similar entities. J Am Acad Dermatol. 1979;1(5):413-421.

3. Chen WT, Hsiao PF, Wu YH. Spectrum and clinical variants of giant cell elastolytic granuloma. Int J Dermatol. 2017;56(7):738-745.

4. Echávez MD, Achenbach RE, Pérez LC, et al. Granuloma anular elastolítico a células gigantes: Comunicación de un caso y comentarios sobre su ubicación nosológica. Rev argent dermatol. 2017;98(3).

5. Arora S, Malik A, Patil C, et al. Annular elastolytic giant cell granuloma: A report of 10 cases. Indian Dermatol Online J. 2015;6(Suppl 1):S17-20.

6. Gutierrez GE, Gomez BS, Alvarez PA, et al. Elastolytic giant cell granuloma: clinic-pathologic review of twenty cases. Dermatol Online J. 2013;19(10):20019.

7. Limas C. The spectrum of primary cutaneous elastolytic granulomas and their distinction from granuloma annulare: A clinicopathological analysis. Histopathology. 2004;44(3):277-282.

8. Gopalakrishnan V, Thankappan TP, Sulochana G. Annular elastolytic giant cell granuloma: An atypical presentation. Indian J Dermatopathol Diagn Dermatol. 2018;5(1):51-53. 